\title{
Zum 10. Mal verliehen: Der Medienpreis der Deutschen Lungenstiftung
}

\author{
Bei der 56. DGP-Jahrestagung wurde zum 10. Mal der Wilhelm \\ und Ingeborg Roloff-Preis (WIR-Preis) der Deutschen \\ Lungenstiftung e. V. vergeben. Dies geschah, nachdem die \\ Jury, der Prof. Harald Morr und Prof. Gerhard Siemon seit vielen \\ Jahren angehören, getagt und zusammen mit 3 weiteren \\ Juroren eine einvernehmliche Entscheidung getroffen hatten. \\ Der Preis für die Jahre 2013/2014 ging an die Journalisten \\ Dimitri Ladischensky, Hamburg, und Christine Matz, Hürth.
}

Mit dem WIR-Preis ausgezeichnet wurden in diesem Jahr Journalisten für ihre herausragenden Beiträgen über Lungenkrankheiten - in diesem Fall zur Mukoviszidose - in der Zeitschrift „mare“ und für „Stern TV/ RTL“. Die Preise wurden durch Prof. Dr. Marek Lommatzsch, Rostock, und Dr. Eckart Roloff, Bonn, überreicht. Benannt ist der Preis nach dem Lungenfacharzt Dr. med. habil. Wilhelm Roloff und dessen Ehefrau Ingeborg.

\section{Mukoviszidose und Meer \\ $\nabla$}

Den 1. Preis, verbunden mit $3000 €$, erhielt Dimitri Ladischensky als Anerkennung für eine äußerst eindrucksvolle Schilderung zum Leben und Leiden zweier Schwestern, die beide an Mukoviszidose erkrankt sind . Seine Reportage „Emmi und Soschka“, veröffentlicht in der August/September-Ausgabe 2014 der Zeitschrift „mare“, fesselt, so sagen die Juroren, dank beträchtlicher Empathie und sehr einfühlsamer Sprache. Der ausführliche Beitrag lässt die Leser mitempfinden, was die beiden jungen Mädchen und vor allem die Mutter, eine Krankenschwester, über Jahre hinweg durch diese Krankheit erleben und wie entscheidend die richtige Therapie ist. Ladischenskys Text geht nahe. Unterstützt wird er durch sehr intensive Bilder der Fotografin Joanna Nottebrock.

Dimitri Ladischensky wurde 1972 in Hamburg geboren. Er studierte Germanistik, Geschichte und Volkswirtschaftslehre in Freiburg, Kopenhagen und Berlin. Nach dem Examen 1998 absolvierte er die Deutsche Journalistenschule in München. Danach schrieb er für „Geo Saison“. Seit Herbst 2001 ist er Leiter der Ressorts Leben und Politik der Zeitschrift „mare“.

\section{Schicksal Lungentransplantation $\nabla$}

Den 2. Preis über $2000 €$ bekam Christine Matz von der Redaktion „Stern TV“. Die Jury erkennt damit ihren musterhaften Fernsehbeitrag zum Thema Mukoviszidose und Lungentransplantation an, den RTL am 19. Juni 2013 ausgestrahlt hatte. Christine Matz hat einen jungen Patienten namens Konstantin Rafflenbeul und dessen Familie 2 Jahre lang begleitet. In Worten und Bildern vermittelt der Film sehr eindringlich, welche Folgen diese Krankheit hat und was 2 Lungentransplantationen im Jahr 2013 für den 14-Jährigen bedeuteten, da sich sein Körper gegen die erste Übertragung wehrte. Inzwischen geht es ihm gut. Konstantin konnte jetzt sogar zusammen mit seiner Mutter zur Jahrestagung nach Berlin kommen. Die Jury belohnt neben dem Fernsehfilm auch das ausführliche Studiogespräch zwischen dem Moderator Steffen Hallaschka und Konstantin, seiner Mutter und dem Lungenfacharzt Dr. Nicolaus Schwenk, Hannover. Für Mitte 2015 plant RTL einen weiteren Film über Konstantin und dessen Entwicklung.

Christine Matz wurde 1968 in Pirmasens geboren. 1997 hat sie ihr Studium der Germanistik und Sportwissenschaft in Saarbrücken abgeschlossen. Während des Studiums war sie für mehrere Redaktionen des Saarländischen Rundfunks in Saarbrücken und für den Mitteldeutschen Rundfunk in Dresden tätig. Nach dem Studium war sie Volontärin in der Lokalredaktion von Sat 1 in Leipzig. Seit Ende 1999 arbeitet Christine Matz als Redakteurin und Reporterin bei Stern TV.

Eckart Klaus Roloff, Bonn 